\title{
杯[4]脯氨酸衍生物的合成及其对扁桃酸对映异构体的手性识别
}

\author{
李正义 ${ }^{a}$ 周 坤 ${ }^{a} \quad$ 来 源 ${ }^{a}$ 孙小强 $*, a$ 王乐勇 $*, b$

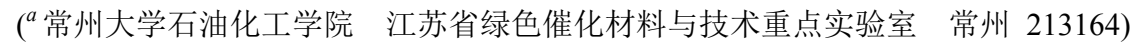 \\ ( ${ }^{b}$ 超分子化学与智能材料实验室 南京大学化学化工学院 南京 210093)
}

\begin{abstract}
摘要 以氨基杯 [4]芳烃为原料, 合成了五种手性杯 [4]脯氨酸衍生物, 其结构经 ${ }^{1} \mathrm{H} N \mathrm{NM},{ }^{13} \mathrm{C} N \mathrm{NM}, \mathrm{IR}, \mathrm{ESI}-\mathrm{MS}$ 及元素 分析表征. 运用核磁共振技术研究它们对扁桃酸对映异构体的手性识别性能, 结果表明 5,11-二脯氨酰胺取代杯 [4]芳烃 2b 对两种不同构型的扁桃酸异构体具有较好的选择性识别作用 $\left(K_{L} / K_{D} \approx 94\right)$. 提出可能的识别机理为分子间多种超分 子相互协同作用, 包括酸碱作用、氢键和 $\pi \cdots \pi$ 堆积作用.
\end{abstract}

关键词＼cjkstart杯[4]芳烃; $L$-脯氨酸; 扁桃酸; 手性识别; 核磁共振

\section{Synthesis of Calix[4]proline Derivatives and Their Chiral Recognition for Enantiomers of Mandelic Acid}

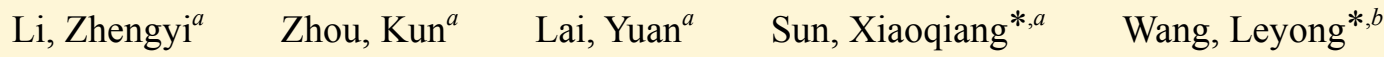 \\ ( ${ }^{a}$ Jiangsu Key Laboratory of Advanced Catalytic Materials and Technology, School of Petrochemical Engineering, \\ Changzhou University, Changzhou 213164) \\ $\left({ }^{b}\right.$ Laboratory for Supramolecular Chemistry and Dynamic Materials, School of Chemistry and Chemical Engineering, \\ Nanjing Universty, Nanjing 210093)
}

\begin{abstract}
Five chiral calix[4]proline derivatives were designed and synthesized from aminocalix[4]arenes, and their structures were characterized by ${ }^{1} \mathrm{H}$ NMR, ${ }^{13} \mathrm{C}$ NMR, IR, ESI-MS and elemental analysis. The chiral recognition ability for enantiomers of mandelic acid was investigated by NMR techniques, and the results showed that 5,11-diprolinamides substituted calix[4]arene $\mathbf{2 b}$ could well and selectively recognize the two enantiomers of mandelic acid $\left(K_{L} / K_{D} \approx 94\right)$. A possible recognition mechanism was proposed, which was constructed by cooperative multi-supramolecular interactions including intermolecular acid-base, hydrogen bond and $\pi \cdots \pi$ stacking interactions.
\end{abstract}

Keywords calix[4]arene; $L$-proline; mandelic acid; chiral recognition; NMR

分子识别一直是超分子化学研究的主要内容之一, 早期经典的例子当属冠醚对碱金属离子的识别 ${ }^{[1]}$. 手性 是自然界的普遍现象，如生物分子大多为手性分子，生 物体内物质的合成及信息的传送也都要求严格的选择 性及方向性, 手性药物在人体内具有不同的作用效果, 因此对手性分子识别的研究有助于解开自然界手性的 秘密, 是分子识别研究的重要内容之一 ${ }^{[2]}$. 扁桃酸 (MA) 是一种广泛应用于药物合成工业的中间体, 它的 两种对映体表现的性质各异 ${ }^{[3]}$. 如 $R$-MA 用于头孢菌类 抗生素侧链修饰剂, $S$-MA 是合成用于治疗尿急、尿频和
尿失禁药物 $S$-奥昔布宁的前体原料, MA 及其衍生物对 映体的纯度会影响相应药物的药效. 因此 MA 的光学拆 分和手性识别研究一直是相关领域的研究热点 ${ }^{[4]}$.

杯芳烃作为继冠醚和环糊精之后的第三代超分子 主体分子, 具有良好的可修饰性和空腔可调节等特点, 在其上沿和下沿可引入各种基团, 能借助诸如氢键、静 电作用、疏水作用以及 $\pi \cdots \pi$ 堆积等非共价键协同作来 识别客体分子 ${ }^{[5]}$. 随着手性杯芳烃的发展, 杯芳烃在手 性分子识别领域的应用已日益广泛 ${ }^{[6]}$, 如郑炎松等将手 性氨基醇嫁接在杯 [4]芳烃的下沿, 实现了对 MA 的手性

\footnotetext{
*E-mail: sunxiaoqiang@yahoo.com, Lywang@nju.edu.cn

Received February 10, 2015; revised March 2, 2015; published online March 19, 2015
}

Project supported by the NSFC (No. 21002009), Major Program for the Natural Science Research of Jiangsu Colleges and Universities (Nos. 12KJA150002, 14KJA150002), Priority Academic Program Development of Jiangsu Higher Education Institution (PAPD), and Qing Lan Project of Jiangsu Province. 国家自然科学基金(No. 21002009)、江苏省高校自然科学研究重大项目(Nos. 12KJA150002, 14KJA150002)、江苏省优势学科和江苏省青蓝工程资助项 目. 
识别 ${ }^{[7]}$. 此外, 固有手性杯芳烃也被成功应用于 MA 的 手性识别 ${ }^{[8]}$. 核磁共振(NMR)技术作为分子识别研究的 最常用手段之一, 已广泛用于对映体的识别和检测 ${ }^{[9]}$, 通过对 NMR 图谱中相关质子的裂分情况和化学位移研 究, 可以直观地观测识别过程中的识别位点和相互作用 等信息, 从而推测可能的结合方式. 我们课题组长期致 力于手性杯芳的合成及应用研究 ${ }^{[10]}$, 本文设计将天然 的脯氨酸引入杯 [4]芳烃的上沿获得一系列手性的杯 [4] 脯氨酸衍生物, 运用 NMR 技术研究它们对 MA 的手性 识别性能.

\section{1 结果与讨论}

\section{1 杯[4]脯氨酸衍生物的合成}

杯芳烃的下沿修饰相对较容易, 而在其上沿修饰较 困难 ${ }^{[5 a]}$. 我们经过探索, 在杯[4]芳烃上沿通过选择性硝 化再还原即可制备五种不同位置和数目的氨基取代杯 [4] 芳烃 ${ }^{[11]}$. 然后将上沿的氨基与 Boc-保护的 $L-\mathrm{MA}$ 在脱 水剂 $N, N^{\prime}$-二环已基碳酰亚胺(DCC)和对二甲氨基吡啶 (DMAP)作用下脱水得酰胺键桥连的Boc-保护的杯 [4]脯 氨酸衍生物中间体, 最后得用三氟乙酸脱保护便可得到 对应的五种手性杯 [4]脯氨酸衍生物 $\mathbf{2 a} \sim 2 \mathbf{e}$ (Scheme 1).
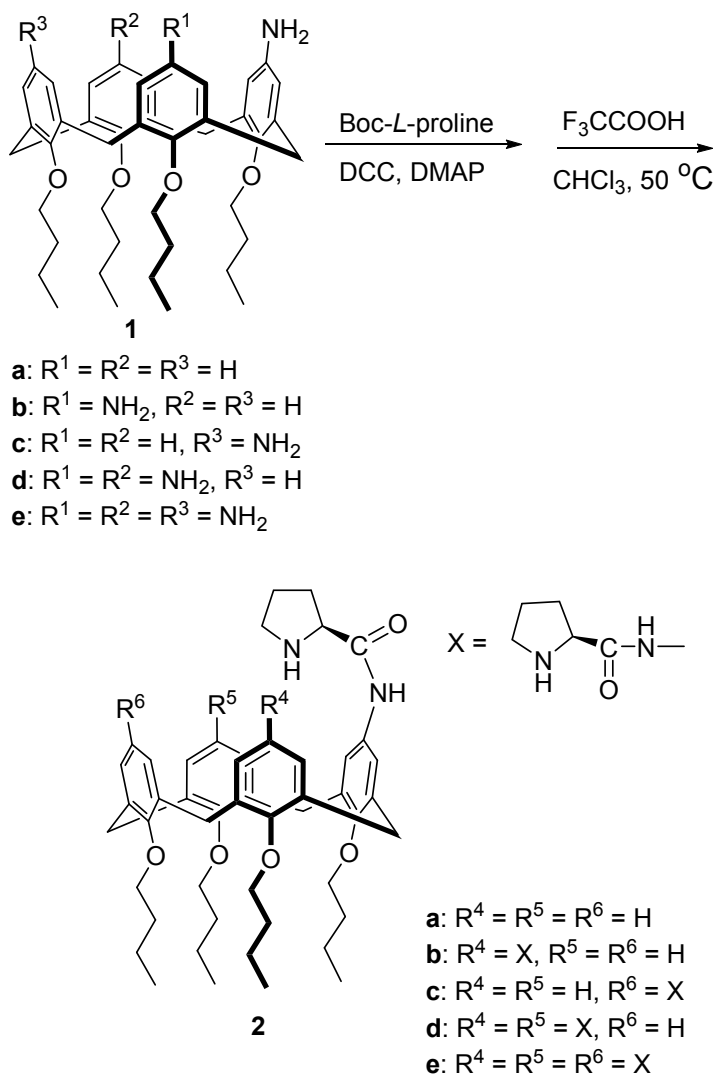

图式 1 杯[4]脯氨酸衍生物的合成

Scheme 1 Synthesis of calix[4]proline derivatives
1.2 杯[4]芳烃脯氨酸衍生物对扁桃酸对映异构体的手 性识别

\subsection{1 主体分子的篮选}

以 MA 外消旋体 $\mathbf{3}$ 为客体分子, 运用核磁共振技术 研究上述杯 [4]脯氨酸衍生物对 MA 对映异构体 $(D-3$ 或 $L-3)$ 的手性识别性能. 首先, 我们考察五种手性杯[4]脯 氨酸衍生物 2a 2 e 对 MA 对映异构体的手性识别能力. 在 $\mathrm{CDCl}_{3}$ 中分别配制 $1: 1$ 的主客体化合物 $(5 \mathrm{mmol} / \mathrm{L})$, 观测 $\mathrm{MA}$ 中 $\mathrm{PhCHO}$ 上的手性质子在 ${ }^{1} \mathrm{H}$ NMR 中的化学 裂分及位移, 笁选出识别效果最佳的主体分子. 结果表 明，5,11-二脯氨酰胺取代杯[4]芳烃 $2 \mathbf{b}$ 表现出了最强的 识别能力, 其对 $D, L-\mathrm{MA}$ 的识别可以达到 $28.5 \mathrm{~Hz}$. 如图 1 所示, 在外消旋 $\mathrm{MA}$ 中 $\mathrm{PhCHO}$ 的 $\mathrm{H}$ 质子在 ${ }^{1} \mathrm{H} \mathrm{NMR}$ 谱中表现为一个单峰, 将主体分子 $\mathbf{2 b}$ 滴加到 MA 的 $\mathrm{CDCl}_{3}$ 溶液后, $\mathrm{MA}$ 中的 $\mathrm{PhCHO}$ 单峰就会裂分为积分面 积相等的两个单峰, 即主体分子 $\mathbf{2 b}$ 对不同构型的 MA 具有选择性识别作用 ${ }^{[7 ~ 9]}$. 当以 5-单脯氨酰胺取代杯 [4] 芳烃 $2 \mathbf{a}$ 作主体时，只发生 $4.2 \mathrm{~Hz}$ 的裂分; 同为二取代的 5,17 -二脯氨酰胺取代杯[4]芳烃 $2 \mathrm{c}$ 却只有 $18 \mathrm{~Hz}$; 当选用 5,11,17-三脯氨酰胺取代杯 [4]芳烃 2d 和 5,11,17,23-四脯 氨酰胺取代杯 [4]芳烃 2e 时, 它们的 ${ }^{1} \mathrm{H}$ NMR 中对应的 位置显示为一个大包，无法分辨，可能是由于太多的分 子内和分子间的氢键作用所致. 由此可见，5,11-二脯氨 酰胺取代杯 [4]芳烃 $\mathbf{2 b}$ 对 $\mathbf{3}$ 的手性识别性能最佳.

\subsection{3 识别机理研究}

固定主体 $\mathbf{2 b}$ 与光学纯的 MA 客体分子的总浓度 $(5$ $\mathrm{mmol} / \mathrm{L}$ )不变，改变主客体间的不同配比，绘制核磁滴 定曲线和 Job 曲线(图 2). 结果表明 $\mathbf{2 b}$ 与 $D-3$ 或 $L-3$ 分 别形成 $1: 2$ 的络合物, 即主客体之间的结合比为 1 : $2^{[7]}$. 因此, 我们推测杯[4]芳烃 $\mathbf{2 b}$ 上两个手性脯氨酰胺 残基分别结合一个 MA 客体分子，但两者之间又同时存 在相互的协同作用，因为同等条件下 5,11-相邻的二脯 氨酰胺取代杯 [4]芳烃 $\mathbf{2 b}$ 对 3 的识别效果比 5,17-相对的 二脯氨酰胺取代杯 [4]芳烃 $2 \mathbf{c}$ 强([2b]：[3]=1: $1, \Delta \delta$ : 0.095 vs. 0.060), 这可能是由于 $2 \mathrm{c}$ 中两个手性脯氨酰胺 功能基团相距较远，空间上与 MA 客体分子不匹配，不 能起到相互协同的作用. 由此, 我们提出了可能的识别 机理(Scheme 2). 首先, $\mathrm{MA}$ 的 $\mathrm{COOH}$ 与 $\mathbf{2 b}$ 中的仲胺通 过酸碱相互识别, 同时 $\mathrm{O}=\mathrm{C}$ 与酰胺 $\mathrm{NH}$ 形成氢键; 其 次, $\mathrm{MA}$ 的 $\mathrm{OH}$ 与 $\mathbf{2 b}$ 中另一分子的脯氨酰胺的 $\mathrm{O}=\mathrm{CN}$ 作用形成氢键，从而使两个脯氨酰胺达到相互协同作用 的功效; 最后，两个 MA 中的苯基相互之间以及它们与 杯芳烃空穴之间存在 $\pi \cdots \pi$ 堆积作用, 进一步增强了主 体 $\mathbf{2 b}$ 对客体 $\mathbf{3}$ 的识别能力. 


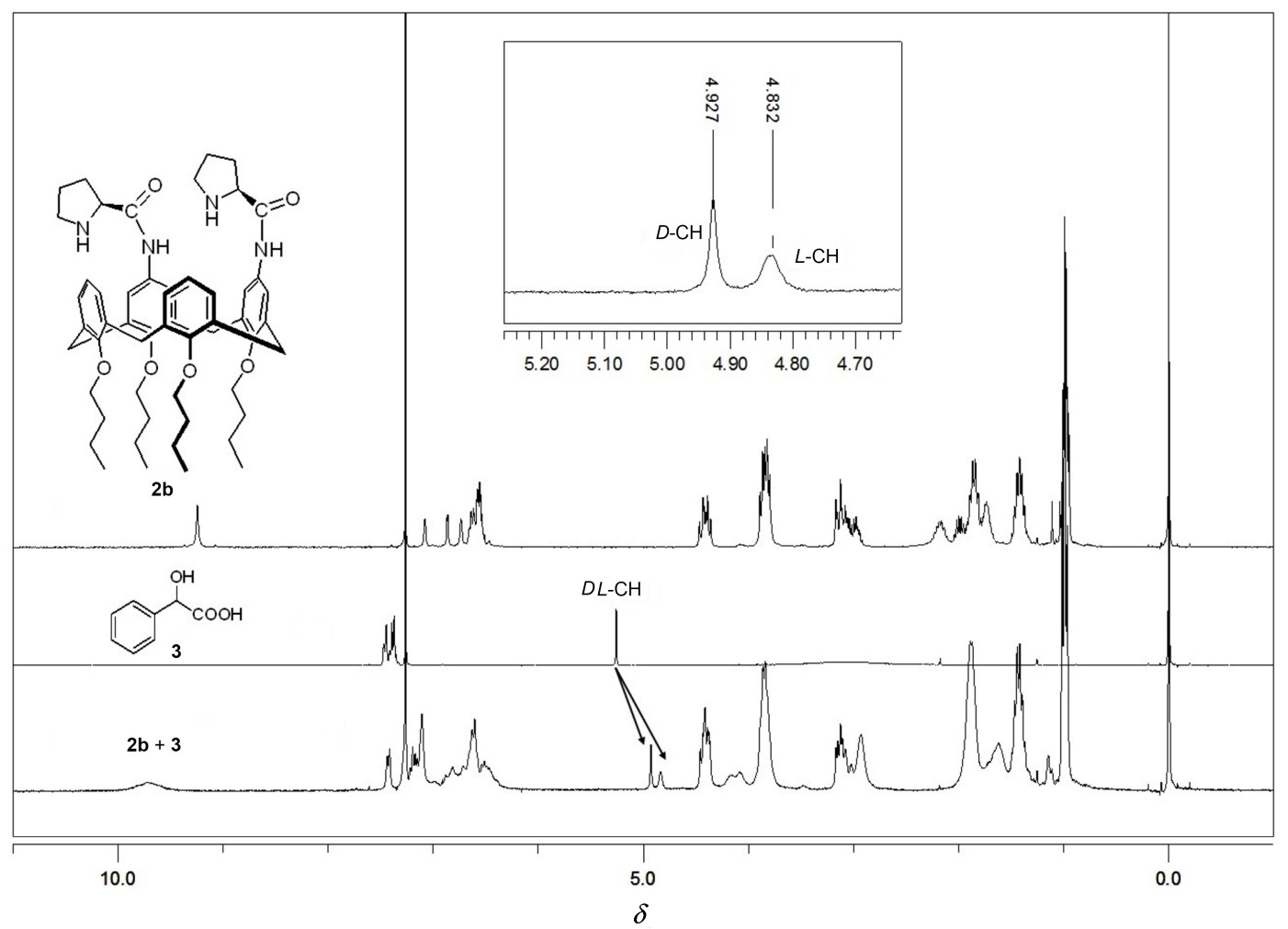

图 1 核磁共振氢谱中 $\mathbf{2 b}$ 对扁桃酸外消旋体 $\mathbf{3}$ 的拆分

Figure 1 The resolution of racemic mandelic acid $\mathbf{3}$ in ${ }^{1} \mathrm{H}$ NMR spectra induced by $\mathbf{2 b}$ $[\mathbf{2 b}]:[3]=1: 1,5 \mathrm{mmol} / \mathrm{L}, \mathrm{CDCl}_{3}$
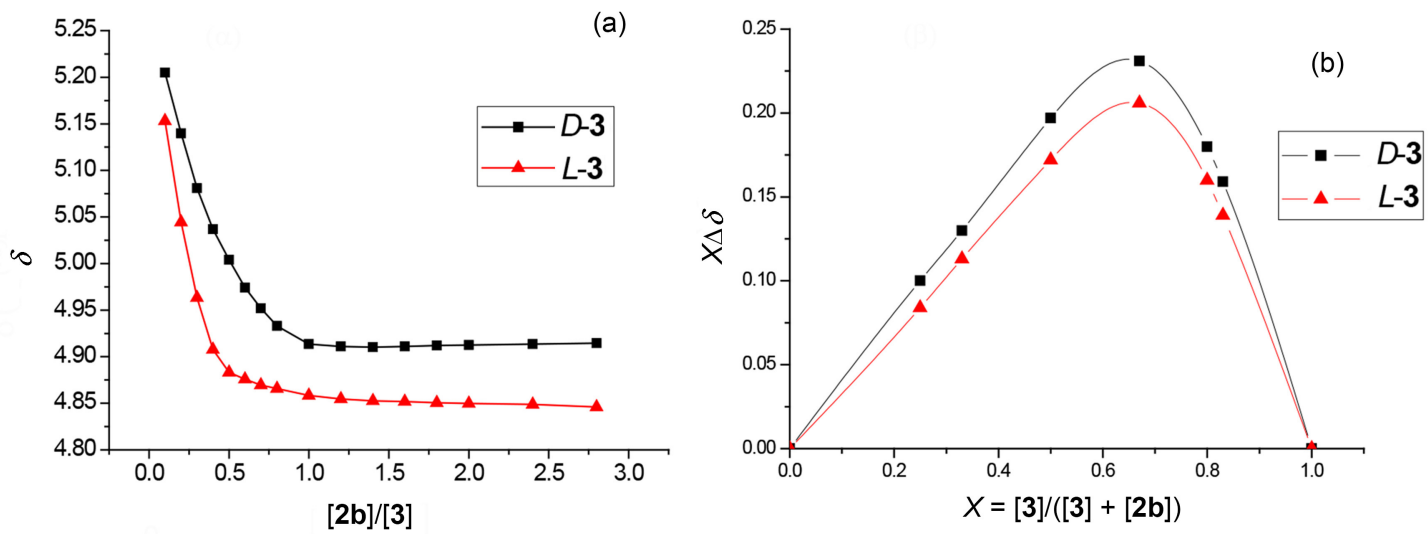

图 $22 \mathbf{b}$ 对光学纯 $D-3$ 和 $L-3$ 的核磁滴定(a)及 Job 曲线(b)

Figure $2{ }^{1} \mathrm{H}$ NMR titration (a) and Job plot (b) of $\mathbf{2 b}$ with optically pure $D-\mathbf{3}$ and $L-\mathbf{3}$

\subsection{4 结合常数计算}

固定光学纯的 $L-\mathbf{3}$ 与 $D-\mathbf{3}$ 的浓度 $(5 \mathrm{mmol} / \mathrm{L})$ 不变, 分别加入不同当量的主体 $\mathbf{2 b}$, 进行核磁滴定. 根据主客 体为 $1: 2$ 的核磁滴定计算结合常数的方法 ${ }^{[12]}$, 分别得 到主体分子 $\mathbf{2 b}$ 与 $L-3$ 与 $D-\mathbf{3}$ 的结合常数(表 1$)$, 进一步 可以得到两者的结合性能之比 $K_{L} / K_{D}=\left(K_{L 1} \times\right.$ $\left.K_{L 2}\right) /\left(K_{D 1} \times K_{D 2}\right) \approx 94$, 即主体分子 $\mathbf{2 b}$ 与 $L-3$ 的结合能力 是 $D-3$ 的 94 倍, 由此可见 5,11 -二脯氨酰胺取代杯 [4]芳 烃 2b 对不同构型的 MA 具有较好的选择性手性识别
表 $12 \mathrm{~b}$ 与扁桃酸对映体的结合常数

Table 1 The association constant of $\mathbf{2 b}$ with enantiomers of mandelic acid

\begin{tabular}{ccl}
\hline & $K_{1} /\left(\mathrm{L} \cdot \mathrm{mmol} /^{-1}\right)$ & $\left.K_{2} /(\mathrm{L} \cdot \mathrm{mmol})^{-1}\right)$ \\
\hline$L-3$ & $3520 \pm 220$ & $1600 \pm 90$ \\
$D-3$ & $350 \pm 50$ & $170 \pm 10$ \\
\hline
\end{tabular}

作用. 


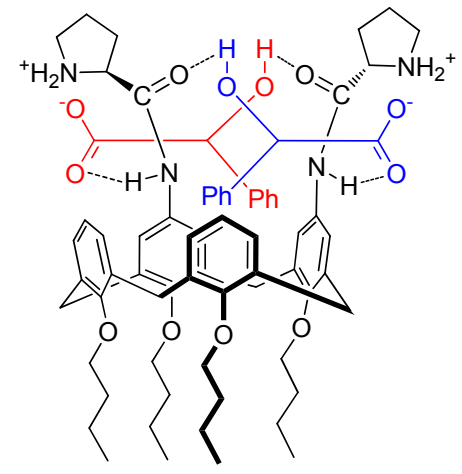

图式 2

Scheme 2

\subsection{5 拆分性能研究}

最后, 我们以 5,11-二脯氨酰胺取代杯[4]芳烃 2b 作 为主体分子, 固定 MA 消旋体 $\mathbf{3}$ 浓度不变, 通过核磁滴 定考察不同浓度的 $\mathbf{2 b}$ 主体对其的手性拆分能力. 如图 3 所示, 只需加入极少量的主体 $\mathbf{2 b}$ 即可引起 $\mathrm{MA}$ 消旋体 $\mathbf{3}$ 中 $\mathrm{PhCHO}$ 上质子的裂分和位移变化, 当主客体物质的 量比([2b]：[3])达到 $2: 1$ 时, MA 外消旋中 $D-3$ 和 $L-3$ 的 $\mathrm{PhCHO}$ 质子的化学位移趋于平稳, 当 $[2 \mathbf{b}]:[3]=4$ : 1 时两者之间的差值达到最大 $\left(\Delta \delta_{\text {max }}=0.099\right)$, 此时, 即 为最佳识别点.

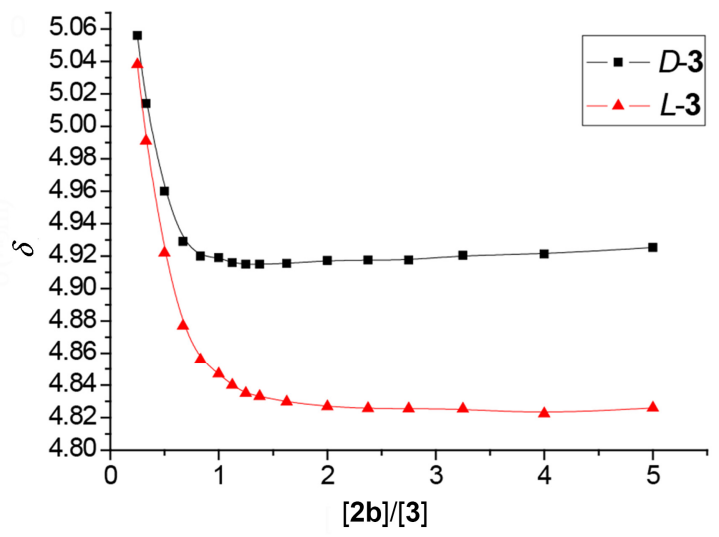

图 $32 \mathrm{~b}$ 对扁桃酸消旋体 $\mathbf{3}$ 的核磁滴定

Figure $3{ }^{1} \mathrm{H}$ NMR titration plot of $\mathbf{2 b}$ with racemic mandelic acid $\mathbf{3}$

\section{2 结论}

本文设计合成了五种不同数目及位置取代的手性 杯[4]脯氨酸衍生物, 运用核磁共振技术研究了它们对 扁桃酸外消旋体的手性识别性能, 笁选出识别能力最强 的主体分子为 5,11-二脯氨酰胺取代杯[4]芳烃 $\mathbf{2 b}$, 运用 核磁滴定测定其与扁桃酸客体分子以 $1: 2$ 结合, 进而 提出可能的多种超分子作用(酸碱作用、氢键、 $\pi \cdots \pi$ 堆 积)相互协同的识别机制. 结合常数计算结果表明 $\mathbf{2 b}$ 优 先与 $L-3$ 的结合, 其结合作用比 $D-3$ 强 94 倍, 即手性杯
[4]脯氨酸 2b 对两种不同构型的扁桃酸异构体具有较好 的选择性识别作用. 该研究将进一步拓宽杯芳烃在手性 分子识别和光学拆分领域的应用.

\section{3 实验部分}

\section{1 仪器与试剂}

XT4-100X 型显微熔点测定仪 (上海申光仪器仪表 有限公司, 未经校正); SHIMADZU LCMS-2020 型质谱 仪(日本); BRUCKER Avance III 300MHz 型核磁共振仪 (德国), ${ }^{1} \mathrm{H}$ 核磁共振频率为 $300 \mathrm{MHz},{ }^{13} \mathrm{C}$ 核磁共振频率 为 $75 \mathrm{MHz}, \mathrm{TMS}$ 为内标; VARIAN FT-IR 8400S 型红外 光谱仪(美国), $\mathrm{KBr}$ 压片.

薄层层析用硅胶、柱层析硅胶(青岛海洋化工公司), 5-氨基-25,26,27,28-四正丁基杯[4]芳烃(1a)参照文献的 方法制备 ${ }^{[11]}$, 其它试剂均为市售分析纯试剂.

\section{2 杯[4]脯氨酸衍生物 $2 a \sim 2 e$ 的合成}

将 5-氨基-25,26,27,28-四正丁基杯[4]芳烃(1a) (0.66 $\mathrm{g}, 1 \mathrm{mmol}$ )溶于 $\mathrm{CH}_{2} \mathrm{Cl}_{2}(50 \mathrm{~mL})$, 向其加入 $L$-Boc-脯氨酸 (0.24 g, $1.1 \mathrm{mmol})$, DCC (0.31 g, $1.5 \mathrm{mmol})$ 和 DMAP (0.0611 g, $0.5 \mathrm{mmol})$. 在室温条件下搅拌 $10 \mathrm{~h}$, 停止反 应, 过滤除去不溶的 DCU, 滤液蒸发浓缩. 采用柱层析 $[V$ (石油醚) $: V$ (乙酸乙酯 $)=8: 1 \sim 3: 1$ ), 得到白色固 体(0.74 g). 紧接着将其溶于 $\mathrm{CHCl}_{3}(30 \mathrm{~mL})$, 滴加三氟 乙酸 $(1 \mathrm{~mL})$, 混合物在 $50{ }^{\circ} \mathrm{C}$ 下反应 $3 \mathrm{~h}$. 停止反应, 蒸 去溶剂, 加入 $\mathrm{CHCl}_{3}(30 \mathrm{~mL})$ 和 $\mathrm{H}_{2} \mathrm{O}(30 \mathrm{~mL})$, 用 $\mathrm{NaOH}$ 溶液 $(2 \mathrm{~mol} / \mathrm{L})$ 调 $\mathrm{pH}$ 至 $8 \sim 9$. 分液, 水层再用 $\mathrm{CHCl}_{3}(30$ $\mathrm{mL})$ 提取一次, 合并有机层, 饱和食盐水洗 $(30 \mathrm{~mL})$, 无 水 $\mathrm{Na}_{2} \mathrm{SO}_{4}$ 干燥. 过滤, 蒸干溶剂得纯的目标化合物 $\mathbf{2 a}$.

化合物 $\mathbf{2 a} \sim 2 \mathrm{e}$ 的合成方法同 $\mathbf{2 a}$, 只需调整相应 $L$-Boc-脯氨酸的用量即可.

5-脯氨酰胺-25,26,27,28-四正丁基杯 [4]芳烃(2a): 白色固体，收率 86\%. m.p. 65 67 ${ }^{\circ} \mathrm{C} ;{ }^{1} \mathrm{H}$ NMR (300 $\left.\mathrm{MHz}, \mathrm{DMSO}-d_{6}\right) \delta: 0.97\left(\mathrm{t}, J=7.5 \mathrm{~Hz}, 12 \mathrm{H}, \mathrm{CH}_{3}\right), 1.37 \sim$ $1.53\left(\mathrm{~m}, 8 \mathrm{H}, \mathrm{CH}_{2}\right), 1.57 \sim 1.76\left(\mathrm{~m}, 4 \mathrm{H}, \mathrm{CH}_{2}\right), 1.80 \sim 1.95$ $\left(\mathrm{m}, 8 \mathrm{H}, \mathrm{CH}_{2}\right), 2.84\left(\mathrm{t}, J=6.5 \mathrm{~Hz}, 2 \mathrm{H}, \mathrm{NCH}_{2}\right), 3.10(\mathrm{~d}, J=$ $\left.13.2 \mathrm{~Hz}, 2 \mathrm{H}, \mathrm{ArCH}_{2} \mathrm{Ar}\right), 3.16(\mathrm{~d}, J=13.2 \mathrm{~Hz}, 2 \mathrm{H}$, ArCH2Ar), 3.56 3.61 (m, 1H, NCHCO), 3.75 3.89 (m, $\left.8 \mathrm{H}, \mathrm{ArOCH}_{2}\right), 4.31$ (d, $\left.J=13.2 \mathrm{~Hz}, 2 \mathrm{H}, \mathrm{ArCH}_{2} \mathrm{Ar}\right), 4.34$ (d, $\left.J=13.2 \mathrm{~Hz}, 2 \mathrm{H}, \mathrm{ArCH}_{2} \mathrm{Ar}\right), 6.45 \sim 6.61$ (m, 7H, ArH), $6.70(\mathrm{~d}, J=7.5 \mathrm{~Hz}, 2 \mathrm{H}, \operatorname{ArH}), 6.96(\mathrm{~d}, J=2.7 \mathrm{~Hz}, 1 \mathrm{H}$, ArH), 7.00 (d, $J=2.7 \mathrm{~Hz}, 1 \mathrm{H}, \mathrm{ArH}), 9.52$ (s, 1H, CONH); ${ }^{13} \mathrm{C}$ NMR (75 MHz, $\mathrm{CDCl}_{3}$ ) $\delta: 14.1,19.3,19.4,25.0,25.8$, 26.3, 26.9, 29.4, 30.7, 31.0, 32.1, 32.2, 32.4, 45.6, 47.3, $51.7,61.0,74.8,74.9,119.7,121.6,121.9,122.0,127.9$, $128.2,128.3,131.6,134.4,134.6,135.8,136.0,136.1$, 
153.5, 156.2, 157.0, 172.6; IR (KBr) v: 3448, 2959, 2930, 2869, 1683, 1601, 1525, 1459, 1382, 1291, 1245, 1087, 1028, $762 \mathrm{~cm}^{-1}$; ESI-MS m/z (\%): $761\left([\mathrm{M}+1]^{+}, 100\right)$, $783\left([\mathrm{M}+\mathrm{Na}]^{+}, 32\right), 799\left([\mathrm{M}+\mathrm{K}]^{+}, 20\right)$. Anal. calcd for $\mathrm{C}_{49} \mathrm{H}_{64} \mathrm{~N}_{2} \mathrm{O}_{5}$ : C 77.33, $\mathrm{H} \mathrm{8.48,} \mathrm{N} \mathrm{3.68;} \mathrm{found} \mathrm{C} 77.48, \mathrm{H}$ $8.35, \mathrm{~N} 3.76$.

5,11 -二脯氨酰胺-25,26,27,28-四正丁基杯 [4]芳烃 (2b): 白色固体, 收率 84\%. m.p. $115 \sim 117{ }^{\circ} \mathrm{C} ;{ }^{1} \mathrm{H}$ NMR (300 MHz, DMSO- $d_{6}$ ) $\delta: 0.96\left(\mathrm{t}, J=7.5 \mathrm{~Hz}, 12 \mathrm{H}, \mathrm{CH}_{3}\right.$ ), $1.35 \sim 1.50\left(\mathrm{~m}, 8 \mathrm{H}, \mathrm{CH}_{2}\right), 1.56 \sim 1.71\left(\mathrm{~m}, 6 \mathrm{H}, \mathrm{CH}_{2}\right)$, $1.79 \sim 1.90\left(\mathrm{~m}, 8 \mathrm{H}, \mathrm{CH}_{2}\right), 1.94 \sim 2.03\left(\mathrm{~m}, 2 \mathrm{H}, \mathrm{CH}_{2}\right), 2.83$ $\left(\mathrm{t}, J=6.5 \mathrm{~Hz}, 4 \mathrm{H}, \mathrm{NCH}_{2}\right), 3.06(\mathrm{~d}, J=13.2 \mathrm{~Hz}, 1 \mathrm{H}$, $\mathrm{ArCH}_{2} \mathrm{Ar}$ ), 3.12 (d, $\left.J=13.2 \mathrm{~Hz}, 2 \mathrm{H}, \mathrm{ArCH}_{2} \mathrm{Ar}\right), 3.17$ (d, $\left.J=13.2 \mathrm{~Hz}, 1 \mathrm{H}, \mathrm{ArCH}_{2} \mathrm{Ar}\right), 3.55 \sim 3.59$ (m, 2H, NCHCO), $3.71 \sim 3.85\left(\mathrm{~m}, 8 \mathrm{H}, \mathrm{ArOCH}_{2}\right), 4.28(\mathrm{~d}, J=13.2 \mathrm{~Hz}, 1 \mathrm{H}$, $\mathrm{ArCH}_{2} \mathrm{Ar}$ ), 4.31 (d, J=13.2 Hz, 2H, $\mathrm{ArCH}_{2} \mathrm{Ar}$ ), 4.34 (d, $\left.J=13.2 \mathrm{~Hz}, 1 \mathrm{H}, \mathrm{ArCH}_{2} \mathrm{Ar}\right), 6.49 \sim 6.66(\mathrm{~m}, 6 \mathrm{H}, \mathrm{ArH})$, $6.81 \sim 6.89$ (m, 3H, ArH), $6.70(\mathrm{~d}, J=2.4 \mathrm{~Hz}, 1 \mathrm{H}, \mathrm{ArH})$, 9.46 (s, 1H, CONH), 9.49 (s, 1H, CONH); ${ }^{13} \mathrm{C}$ NMR (75 $\left.\mathrm{MHz}, \mathrm{CDCl}_{3}\right) \delta: 14.1,19.3,25.0,26.2,30.7,31.0,31.1$, $32.2,32.3,34.0,47.3,61.0,74.8,119.6,119.8,119.9$, $121.6,121.7,128.1,128.3,131.5,131.6,135.0,135.1$. $135.2,135.3,135.6,135.7,153.3,153.4,156.7,172.6$; IR (KBr) v: 3443, 2959, 2930, 2869, 1677, 1602, 1528, 1463, 1382, 1289, 1242, 1211, 1085, 1029, 870, $765 \mathrm{~cm}^{-1}$; ESI-MS $m / z(\%): 874\left([\mathrm{M}+1]^{+}, 42\right), 896\left([\mathrm{M}+\mathrm{Na}]^{+}\right.$, 100). Anal. calcd for $\mathrm{C}_{54} \mathrm{H}_{72} \mathrm{~N}_{4} \mathrm{O}_{6}$ : C 74.28, H 8.31, N 6.42; found C 74.41, H 8.22, N 6.33 .

5,17-二脯氨酰胺-25,26,27,28-四正丁基杯 [4]芳烃 (2c): 白色固体, 收率 83\%. m.p. 104 106 ${ }^{\circ} \mathrm{C} ;{ }^{1} \mathrm{H}$ NMR (300 MHz, DMSO- $d_{6}$ ) $\delta$ : $0.99\left(2 \mathrm{t}, J=7.2 \mathrm{~Hz}, 12 \mathrm{H}, \mathrm{CH}_{3}\right.$ ), $1.27 \sim 1.35\left(\mathrm{~m}, 4 \mathrm{H}, \mathrm{CH}_{2}\right), 1.50 \sim 2.08\left(\mathrm{~m}, 20 \mathrm{H}, \mathrm{CH}_{2}\right), 2.88$ (t, $\left.J=6.5 \mathrm{~Hz}, 4 \mathrm{H}, \mathrm{NCH}_{2}\right), 3.10$ (d, $J=12.9 \mathrm{~Hz}, 4 \mathrm{H}$, $\left.\mathrm{ArCH}_{2} \mathrm{Ar}\right), 3.62 \sim 3.70\left(\mathrm{~m}, 6 \mathrm{H}, \mathrm{NCHCO}+\mathrm{ArOCH}_{2}\right), 3.93$ (t, $\left.J=7.8 \mathrm{~Hz}, 4 \mathrm{H}, \mathrm{ArOCH}_{2}\right), 4.31$ (d, $J=12.9 \mathrm{~Hz}, 4 \mathrm{H}$, $\mathrm{ArCH}_{2} \mathrm{Ar}$ ), 6.32 (s, 6H, ArH), 7.31 (d, J=3.6 Hz, 4H, $\mathrm{ArH}), 9.73$ (s, 2H, CONH); ${ }^{13} \mathrm{C} \mathrm{NMR}\left(75 \mathrm{MHz}, \mathrm{CDCl}_{3}\right) \delta$ : $14.0,14.2,19.1,19.6,25.0,25.6,26.3,30.7,31.0,31.9$, $32.4,32.5,34.0,47.4,49.0,61.0,74.8,74.9,119.8,119.9$, $122.1,127.5,131.6,133.2,137.1,154.2,155.3172 .7$; IR (KBr) v: 3438, 2959, 2930, 2868, 1679, 1603, 1526, 1461, 1243, 1214, 1085, 1027, 875, $761 \mathrm{~cm}^{-1}$; ESI-MS m/z (\%): $874\left([\mathrm{M}+1]^{+}, 31\right), 896\left([\mathrm{M}+\mathrm{Na}]^{+}, 100\right), 912\left([\mathrm{M}+\mathrm{K}]^{+}\right.$, 18). Anal. calcd for $\mathrm{C}_{54} \mathrm{H}_{72} \mathrm{~N}_{4} \mathrm{O}_{6}$ : C 74.28, H 8.31, N 6.42; found C 74.39, H 8.24, N 6.31.
5,11,17-三脯氨酰胺-25,26,27,28-四正丁基杯 [4]芳 烃(2d): 黄色固体, 收率 70\%. m.p. $148 \sim 150{ }^{\circ} \mathrm{C} ;{ }^{1} \mathrm{H}$ NMR $\left(300 \mathrm{MHz}, \mathrm{DMSO}-d_{6}\right) \delta: 0.93 \sim 1.00\left(\mathrm{~m}, 12 \mathrm{H}, \mathrm{CH}_{3}\right)$, $1.10 \sim 1.38\left(\mathrm{~m}, 6 \mathrm{H}, \mathrm{CH}_{2}\right), 1.47 \sim 1.76\left(\mathrm{~m}, 14 \mathrm{H}, \mathrm{CH}_{2}\right)$, $1.81 \sim 1.92\left(\mathrm{~m}, 8 \mathrm{H}, \mathrm{CH}_{2}\right), 2.78 \sim 2.90\left(\mathrm{~m}, 6 \mathrm{H}, \mathrm{NCH}_{2}\right), 3.06$ (d, $J=13.5 \mathrm{~Hz}, 2 \mathrm{H}, \mathrm{ArCH}_{2} \mathrm{Ar}$ ), 3.14 (d, $J=13.5 \mathrm{~Hz}, 2 \mathrm{H}$, $\left.\mathrm{ArCH}_{2} \mathrm{Ar}\right), 3.50 \sim 3.55(\mathrm{~m}, 1 \mathrm{H}, \mathrm{NCHCO}), 3.62 \sim 3.75(\mathrm{~m}$, $\left.6 \mathrm{H}, \mathrm{NCHCO}+\mathrm{ArOCH}_{2}\right), 3.87 \sim 3.92\left(\mathrm{~m}, 4 \mathrm{H}, \mathrm{ArOCH}_{2}\right)$, $4.28\left(\mathrm{~d}, J=13.5 \mathrm{~Hz}, 2 \mathrm{H}, \mathrm{ArCH}_{2} \mathrm{Ar}\right), 4.36(\mathrm{~d}, J=13.5 \mathrm{~Hz}$, $\left.2 \mathrm{H}, \mathrm{ArCH}_{2} \mathrm{Ar}\right), 6.35 \sim 7.32(\mathrm{~m}, 9 \mathrm{H}, \mathrm{ArH}), 9.28(\mathrm{~s}, 1 \mathrm{H}$, $\mathrm{CONH}), 9.67$ (s, 2H, CONH); ${ }^{13} \mathrm{C} \mathrm{NMR}(75 \mathrm{MHz}$, DMSO- $\left.d_{6}\right) \delta: 14.3,14.4,19.2,19.5,24.9,25.8,26.4,30.8$, $31.0,32.0,32.4,33.8,47.2,48.0,61.0,61.2,74.8,75.0$, $119.3,119.9,120.1,122.2,127.9,132.9,133.8,135.7$, 136.0, 152.0, 153.1, 155.8, 157.1, 172.5, 173.0; IR (KBr) $v: 3324,2958,2930,2873,1697,1672,1627,1604,1551$, $1478,1402,1366,1243,1212,1163,1124,1087,1032$, 981, 877, $772 \mathrm{~cm}^{-1}$; ESI-MS m/z (\%): $986\left([\mathrm{M}+1]^{+}, 52\right)$, $1008\left([\mathrm{M}+\mathrm{Na}]^{+}, 100\right)$. Anal. calcd for $\mathrm{C}_{59} \mathrm{H}_{80} \mathrm{~N}_{6} \mathrm{O}_{7}: \mathrm{C}$ 71.92, H 8.18, N 8.53; found C 71.81, H 8.24, N 8.42.

$5,11,17,23$-四脯氨酰胺-25,26,27,28-四正丁基杯 [4] 芳烃(2e): 黄色固体, 收率 65\%. m.p. 179 $181{ }^{\circ} \mathrm{C} ;{ }^{1} \mathrm{H}$ NMR (300 MHz, DMSO- $\left.d_{6}\right) \delta: 0.97$ (t, $J=7.2 \mathrm{~Hz}, 12 \mathrm{H}$, $\left.\mathrm{CH}_{3}\right), 1.35 \sim 1.48\left(\mathrm{~m}, 8 \mathrm{H}, \mathrm{CH}_{2}\right), 1.57 \sim 1.70(\mathrm{~m}, 12 \mathrm{H}$, $\left.\mathrm{CH}_{2}\right), 1.84 \sim 2.01\left(\mathrm{~m}, 12 \mathrm{H}, \mathrm{CH}_{2}\right), 2.82(\mathrm{t}, J=6.5 \mathrm{~Hz}, 8 \mathrm{H}$, $\mathrm{NCH}_{2}$ ), 3.11 (d, $\left.J=12.9 \mathrm{~Hz}, 4 \mathrm{H}, \mathrm{ArCH}_{2} \mathrm{Ar}\right), 3.53 \sim 3.58$ (m, 4H, NCHCO), 3.81 (t, $J=7.5 \mathrm{~Hz}, 8 \mathrm{H}, \mathrm{ArOCH}_{2}$ ), 4.32 (d, $\left.J=12.9 \mathrm{~Hz}, 4 \mathrm{H}, \operatorname{ArCH}_{2} \mathrm{Ar}\right), 7.00$ (d, $J=2.1 \mathrm{~Hz}, 4 \mathrm{H}$, ArH), 7.06 (d, $J=2.1 \mathrm{~Hz}, 4 \mathrm{H}, \mathrm{ArH}), 9.54$ (s, 4H, CONH); ${ }^{13} \mathrm{C}$ NMR $\left(75 \mathrm{MHz}, \mathrm{CDCl}_{3}\right) \delta: 13.9,14.1,19.3,25.0,26.2$, 30.6, 31.1, 31.2, 32.1, 34.0, 47.3, 49.0, 61.0, 75.1, 119.9, 131.8, 134.9, 135.0, 153.1, 172.9; IR (KBr) v: 3292, 2958, 2931, 2869, 1668, 1602, 1529, 1469, 1416, 1292, 1213, $1143,1105,1069,1029,980,865 \mathrm{~cm}^{-1}$; ESI-MS $m / z(\%)$ : $1098\left([\mathrm{M}+1]^{+}, 16\right), 1120\left([\mathrm{M}+\mathrm{Na}]^{+}, 100\right)$. Anal. calcd for $\mathrm{C}_{64} \mathrm{H}_{88} \mathrm{~N}_{8} \mathrm{O}_{8}$ : C 70.04, $\mathrm{H} \mathrm{8.08,} \mathrm{N} \mathrm{10.21;} \mathrm{found} \mathrm{C} \mathrm{70.16,}$ H 7.99, N 10.14.

\section{3 识别性能测试方法}

\subsubsection{Job 曲线的绘制}

用 $\mathrm{CDCl}_{3}$ 分别配制主体 $\mathbf{2 b}(5 \mathrm{mmol} / \mathrm{L})$ 和客体光学 纯 $L-3(5 \mathrm{mmol} / \mathrm{L})$ 溶液, 将不同体积比的主/客体在核磁 管中混合，总浓度保持 $5 \mathrm{mmol} / \mathrm{L}$ 不变. 按照 $[\mathbf{2 b}] /[L-\mathbf{3}]$ $(V: V, \mu \mathrm{L} / \mu \mathrm{L})(\mathrm{mol} / \mathrm{mol})=300 / 100(3: 1), 300 / 150(2:$ 1), 300/300 (1:1), 180/360 (1:2), 120/360 (1:3), 90/360 (1：4), 70/350 (1：5)共配制 7 份试样，混匀后室 
温下分别测定各个配比时样品的 ${ }^{1} \mathrm{H}$ NMR, 并绘制 Job 曲线(对光学纯 $D-3$ 的滴定采用相同方法).

\subsection{2 主体 $\mathbf{2 b}$ 对光学纯扁桃酸异构体的核磁滴定}

用 $\mathrm{CDCl}_{3}$ 分别配制 $2 \mathbf{b}(50 \mathrm{mmol} / \mathrm{L})$ 和 $L-3$ (10 $\mathrm{mmol} / \mathrm{L})$ 溶液, 在一根核磁管中固定 $L-3$ 体积不变 $(480$ $\mu \mathrm{L})$, 逐渐向其中滴加 $\mathbf{2 b}$ 溶液, 加入体积分别为 9.6, 19.2, 28.8, 38.4, 48, 57.6, 67.2, 76.8, 96, 115.2, 134.4, $153.6,172.8,192,230.4,268.8 \mu \mathrm{L}$, 此时对应的 $[\mathbf{2 b}] /[L-3](\mathrm{mol} / \mathrm{mol})=0.1,0.2,0.3,0.4,0.5,0.6,0.7,0.8$, $1,1.2,1.4,1.6,1.8,2.0,2.4,2.8$, 混匀后室温下分别测定 各个配比时样品的 ${ }^{1} \mathrm{H}$ NMR (对 D-3 的核磁滴定采用相 同方法).

\subsection{3 主体 $\mathbf{2 b}$ 对扁桃酸消旋体的核磁滴定}

用 $\mathrm{CDCl}_{3}$ 分别配制主体 $\mathbf{2 b}(50 \mathrm{mmol} / \mathrm{L})$ 和客体分子 扁桃酸消旋体 $3(10 \mathrm{mmol} / \mathrm{L})$ 溶液, 在一根核磁管中固定 $3(480 \mu \mathrm{L})$ 不变, 逐渐向其中滴加 $\mathbf{2 b}$ 溶液, 加入体积分 别为 $24,32,48,64,80,96,108,120,132,156,192,228$, $264,312,384,480 \mu \mathrm{L}$, 此时对应的 $[\mathbf{2 b}] /[3](\mathrm{mol} / \mathrm{mol})=$ $1: 4,1: 3,1: 2,1: 1.5,1: 1.2,1: 1,1.125: 1,1.25:$ $1,1.375: 1,1.625: 1,2: 1,2.375: 1,2.75: 1,3.25: 1$, $4: 1,5: 1$, 混匀后室温下分别测定各个配比时样品的 ${ }^{1} \mathrm{H}$ NMR.

辅助材料(Supporting Information) 主体分子的篮选、 2b 对 $L-3$ 和 $D-3$ 的核磁滴定谱图及结合常数的计算方 法. 这些材料可以免费从本刊网站 (http://sioc-journal. $\mathrm{cn} /$ )上下载.

\section{References}

[1] Zheng, B.; Wang, F.; Dong, S. Y.; Huang, F. H. Chem. Soc. Rev. 2012, 41,1621 .

[2] (a) Han, C. P.; Hou, X.; Zhang, H. C.; Guo, W.; Li, H. B.; Jiang, L. J. Am. Chem. Soc. 2011, 133, 7644.

(b) Jin, Q. X.; Li, J.; Li, X. G.; Zhang, L.; Fang, S. M.; Liu, M. H. Prog. Chem. 2014, 26, 919 (in Chinese).

(靳清贤, 李晶, 李孝刚, 张莉, 方少明, 刘鸣华, 化学进展, 2014, 26, 919.)

(c) Yang, C. Chin. Chem. Lett. 2013, 24, 437.

[3] Tang, K. W.; Yi, J. M.; Huang, K. L.; Zhang, G. L. Chirality 2009,
21,390 .

[4] (a) Ma, F. N.; Ai, L.; Shen, X. M.; Zhang, C. Org. Lett. 2007, 9, 125 .

(b) Wang, W. G.; Shen, X. M.; Ma, F. N.; Li, Z. J.; Zhang, C. Tetrahedron: Asymmetry 2008, 10, 1193.

(c) Wang, W. G.; Shen, X. M.; Zhang, C. Chin. J. Org. Chem. 2010, 30, 1126 (in Chinese).

(王文革，申秀民，张聪，有机化学，2010, 30, 1126.)

(d) Liu, F. L.; Li, Y. Y.; Wang, W. G.; Xu, J.; Liu, Y. P.; Xiao, Q. B. Chin. J. Org. Chem. 2011, 31, 747 (in Chinese).

(刘丰良, 李媛媛, 王文革, 徐军, 刘言萍, 肖清波, 有机化学, 2011, 31, 747.)

[5] (a) Böhmer, V. Angew. Chem., Int. Ed. 1995, 34, 713.

(b) Ikeda, A.; Shinkai, S. Chem. Rev. 1997, 97, 1713.

(c) Harrowfield, J. Chem. Commun. 2013, 1578.

(d) Casnati, A. Chem. Commun. 2013, 6827.

(e) Kim, J. S.; Quang, D. T. Chem. Rev. 2007, 107, 3780.

(f) Guo, D. S.; Liu, L. Chem. Soc. Rev. 2012, 41, 5907.

(g) Yuan, Z.; Liang, F. Curr. Org. Chem. 2014, 18, 2016.

(h) Li, L.; Qi, W. G.; Wang, C.; Yan, C. G. Chin. J. Org. Chem. 2013, 33, 1804 (in Chinese).

(李亮, 戚伟光, 王超, 颜朝国, 有机化学, 2013, 33, 1804.)

[6] (a) Qing, G. Y.; Liu, S. Y.; He, Y. B. Prog. Chem. 2008, 20, 1933 (in Chinese).

(卿光炎, 刘顺英, 何永炳, 化学进展, 2008, 20, 1933.)

(b) Mutihac, L.; Lee, J. H.; Kim, J. S.; Vicens, J. Chem. Soc. Rev. 2011, 40, 2777.

[7] (a) Zheng, Y. S.; Zhang, C. Org. Lett. 2004, 6, 1189.

(b) Zheng, Y. S.; Xiao, Q. Chin. J. Chem. 2005, 23, 1289.

(c) Liu X. X.; Zheng, Y. S. Tetrahedron Lett. 2006, 47, 6357.

[8] Shirakawa, S.; Moriyama, A.; Shimizu, S. Org. Lett. 2007, 9, 3177.

[9] Liu, L.; Kou, Y. H.; Wang, L. Y.; Cao, D. R. Chin. J. Org. Chem. 2011, 31, 964 (in Chinese).

(刘陆智, 寇玉辉, 汪凌云, 曹德榕, 有机化学, 2011, 31, 964.)

[10] (a) Li, Z. Y.; Chen, J. W.; Wang, L. Y.; Pan, Y. Synlett 2009, 2356.

(b) Li, Z. Y.; Teng, M. Y.; Ma, J. J.; Huang, J.; Wang, L. Y.; Pan, Y. Sci. China, Ser. B: Chem. 2009, 52, 497.

(c) Li, Z. Y.; Lu, C. X.; Huang, G. L.; Ma, J. J.; Sun, H. S.; Wang, L. Y.; Pan, Y. Lett. Org. Chem. 2010, 7, 461.

(d) Li, Z. Y.; Xing, H. J.; Huang, G. L.; Sun, X. Q.; Jiang, J. L.; Wang, L. Y. Sci. Chin. Chem. 2011, 54, 1726.

(e) Li, Z. Y.; Chen, J. W.; Liu, Y.; Xia, W.; Wang, L. Y. Curr. Org. Chem. 2011, 15, 39.

[11] Li, Z. Y.; Ma, J. J.; Chen, J. W.; Pan, Y.; Jiang, J. L.; Wang, L. Y. Chin. J. Chem. 2009, 27, 2031.

[12] (a) Huang, F. H.; Fronczek, F. R.; Gibson, H. W. J. Am. Chem. Soc. 2003, 125, 9272 .

(b) Cheng, P. N.; Lin, C. F.; Liu, Y. H.; Lai, C. C.; Peng, S. M.; Chiu, S. H. Org. Lett. 2006, 8, 435. 